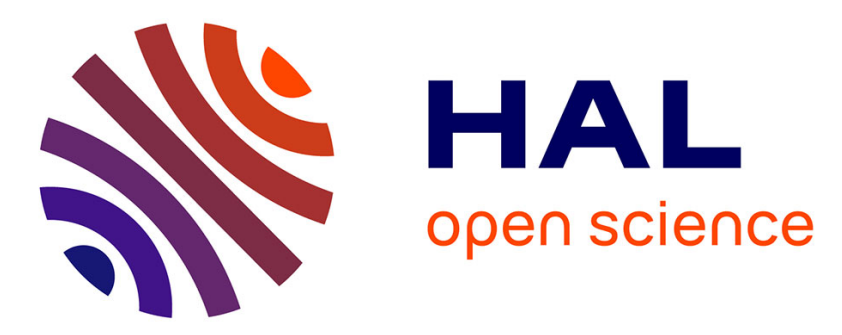

\title{
A 1D model of leukocyte adhesion coupling bond dynamics with blood velocity
}

Bérénice Grec, Bertrand Maury, Nicolas Meunier, Laurent Navoret

\section{To cite this version:}

Bérénice Grec, Bertrand Maury, Nicolas Meunier, Laurent Navoret. A 1D model of leukocyte adhesion coupling bond dynamics with blood velocity. Journal of Theoretical Biology, 2018, 452, pp.35-46. 10.1016/j.jtbi.2018.02.021. hal-01566770

\section{HAL Id: hal-01566770 \\ https://hal.science/hal-01566770}

Submitted on 21 Jul 2017

HAL is a multi-disciplinary open access archive for the deposit and dissemination of scientific research documents, whether they are published or not. The documents may come from teaching and research institutions in France or abroad, or from public or private research centers.
L'archive ouverte pluridisciplinaire HAL, est destinée au dépôt et à la diffusion de documents scientifiques de niveau recherche, publiés ou non, émanant des établissements d'enseignement et de recherche français ou étrangers, des laboratoires publics ou privés. 


\title{
A 1D model of leukocyte adhesion coupling bond dynamics with blood velocity
}

\author{
Bérénice Grec $^{\mathrm{a}}$, Bertrand Maury ${ }^{\mathrm{b}}$, Nicolas Meunier ${ }^{\mathrm{a}}$, Laurent Navoret $^{\mathrm{c}}$ \\ ${ }^{a}$ MAP5, UMR CNRS 8145, Université Paris Descartes, Sorbonne Paris Cité, 45 rue des \\ Saints Pères, 75270 Paris Cedex 6, France, email: berenice.grec@parisdescartes.fr, \\ nicolas.meunier@parisdescartes.fr \\ ${ }^{b}$ Laboratoire de Mathématiques d'Orsay, Université Paris-Sud, 91405 Orsay Cedex, \\ France, email: bertrand.maury@math.u-psud.fr \\ ${ }^{c}$ IRMA, UMR 7501, Université de Strasbourg et CNRS, 7 rue René Descartes, 67000 \\ Strasbourg, France, email: laurent.navoret@math.unistra.fr
}

\begin{abstract}
Cell adhesion on the vascular wall is a highly coupled process where blood flow and adhesion dynamics are closely linked. Cell dynamics in the vicinity of the vascular wall is driven mechanically by the competition between the drag force of the blood flow and the force exerted by the bonds created between the cell and the wall. Bonds exert a friction force. Here, we propose a mathematical model of such a competitive system, namely leukocytes whose capacity to create bonds with the vascular wall and transmigratory ability are coupled by integrins and chemokines. The model predicts that this coupling gives rise to a dichotomic cell dynamic, whereby cells switch from rolling to firm arrest, through non linear effects. Cells can then transmigrate through the wall. These predicted dynamic regimes are compared to in-vitro trajectories of leukocytes. We expect that competition between friction and drag force in particle dynamics (such as shear stress-controlled nanoparticle capture) can lead to similar dichotomic mode.
\end{abstract}

Keywords:

\section{Introduction}

Cell transmigration is a crucial process during many physiological and pathological events, such as inflammation, cancer metastasis or atherosclerosis. Cell transmigration is a highly integrated multistep mechanism comprising three well-defined steps, namely initial contact between the cell and the 
wall, cellwall adhesion and finally firm adhesion leading to cell transmigration. The molecular events orchestrating cell transmigration are still largely unknown. Nonetheless, some key regulatory molecules involved in cell adhesion have now been identified Shamri et al. (2005); Schwarz and Alon (2004). Adhesion requires the creation of bonds between the circulating cell and endothelial cells which counterbalance the drag force of the blood flow. Such knowledge has allowed for cell adhesion events to be both experimentally and mathematically reconstructed and further elucidated.

Leukocyte adhesion has been widely studied experimentally. The nature of the bonds (slip and/or catch) has been the source of active debate during the last decades (Bell (1978) for slip bond description, Finger et al. (1996) for catch bond and see Korn (2007); Ley et al. (2007); Helms et al. (2016) and references therein for more details).

Leukocytes are transported by the blood flow and penetrate the vascular wall according to the so-called adhesion cascade. After an initial contact between the leukocyte and the endothelial cells (capture step), receptor-ligands bonds are formed. The fast association and dissociation rates of these bonds lead to the rolling step: new bonds form at the leukocyte front while old bonds rupture at the rear of the leukocyte. This makes the leukocytes roll on the endothelial wall. During rolling, due to the velocity decrease, leucocytes may detect molecular infection signals such as chemokines (ICAM). Integrins are then activated on its membrane and enable leukocytes to build stronger bonds with the endothelial cells. This leads to the firm adhesion of leukocytes and then finally to their transmigration through the arterial wall. This final step involves the deformation of the cell.

Several models have been proposed to describe cell rolling and adhesion phenomena. In Hammer and Lauffenburger (1987); Hammer and Apte (1992), the leukocyte is modelled as a ligand-coated rigid sphere flowing near a wall. In other works, the ligand-receptor binding follows a chemical kinetic dynamics Bell (1978); Bell et al. (1984). Bonds then exert elastic forces on the sphere while the linear shear flow exerts a hydrodynamic force and torque. In Korn and Schwarz (2008), the Brownian motion of the sphere is taken into account in order to model the spatial receptor-ligand encountering in more details. In the absence of fluid flow, macroscopic models have been developed for cell adhesion force (see Preziosi and Vitale (2011)). In our approach, bonds are not described individually but as a distribution function. This bonds distribution follows a maturation-rupture equation (also called renewal equation) as in Oelz and Schmeiser (2010). In the limit of large 
ligands binding turnover, a friction coefficient can be computed Preziosi and Vitale (2011); Milisic and Oelz (2011).

In this paper, we propose a minimal 1D model to quantify favorable conditions of adhesion in terms of the flow properties (shear stress, viscosity, etc.) and the involved biochemical factors (adhesive molecule density, ligands elasticity, etc.). The particle is described as a point convected by the fluid flow and interacting with the endothelium. The endothelium is assimilated to a straight line. Finally, the fluid velocity is assumed to be directed along the endothelium. At the level of the individual receptor molecule, ligand binding and dissociation are stochastic processes (Poisson processes). The modeling parameters are the bond association-dissociation rates and the bond life-time distribution.

One of the outstanding aims of binding models is to analyse the mechanism of interaction of ligands with the receptor alone, and in combination. Such information provides an important contribution to better understand how an individual cell would potentially react once exposed to a stimulus (inflammation). Therefore, in a second step we are interested in averaging the model based on Poisson point process. Averaging this stochastic model leads to a deterministic Volterra integro-differential equation similar to the one considered in Preziosi and Vitale (2011); Milisic and Oelz (2011). The deterministic one-dimensional model provides the particle location.

The main advantage of the model we present here is to incorporate few adjustable parameters. More precisely, the convection force depends on the Rayleigh friction force and on the convection velocity, whereas the bonds force depends on the bond association/dissociation rates and on the strength of receptorligand interactions. Introducing a scaling of the parameters and variables, we obtain a model with only one scalar parameter. Therefore, this model can be confronted with data in order to estimate cell adhesion.

We first consider a model in which bond forces are described by linear elastic force and in which ligand binding and dissociation rates are constant. After averaging, we obtain a linear Volterra integro-differential equation. Such a linear model is not satisfactory. Indeed it has been observed that the penetration of leukocytes is strongly dependent on the shear flow.

Besides, it has been observed for a long time that bonds life-time may decrease as they are under tension: such bonds are called slip bonds Bell (1978). However, in low stress conditions, it has also been observed that some bonds have the opposite behavior: they become stronger (their lifetime increases) as they are stressed Finger et al. (1996). Such bonds are 
called catch bonds. This latter phenomenon leads to the shear threshold effect: efficient adhesion occurs at sufficiently high shear stress.

In order to incorporate such phenomena, we enrich the stochastic linear model in two different directions. We consider force-dependent bond rates as in Bell (1978). We also consider non-linear elastic laws: we introduce a maximal length of the bonds or we suppose that there is a saturation bound on the bond force. For each of these enriched models we associate an averaged model. In a first step, we verify the relevance of the averaged model by numerically checking its agreement with the stochastic model. In a second step we numerically study the deterministic model. In the case where the model relies on a non-linear elastic law, we observe that there exists a threshold on the convective velocity under which the cell velocity vanishes (the cell stops) and above which the cell is weakly slowed down. Each non-linear model leads to a different threshold velocity which depends on physical parameters (maximal elongation, maximal bond force,...). The model with force-dependent bond rates lead to a similar dichotomic behavior when considering particular force-dependent bond rates. The same model, with other force-dependent bond rates, can also be used to model in a simple way the behavior of catch bonds and the shear threshold effect.

Last, we aim at comparing the results of the models with data of Alon et al. (1997); Korn and Schwarz (2008). We check that the obtained threshold velocities can be effective criteria for adhesion. Selective adhesion indeed requires that the threshold value lies in the range of the physiological flow. We show that the only relevant threshold value is the one coming from the maximal bond elongation model. However due to the large range of the parameters (especially for the association rate that can vary with a factor 100), the comparison with experimental data should be carried out more precisely with specific experiments.

The outline of this article is the following. In Section 2, we present the details of the linear model as well as its long-time asymptotic behavior, recovering friction dynamics. In Section 3, we introduce non-linear versions of the model and we exhibit the dichotomic behaviors. We investigate the influence of two important parameters of the model on cell speed: (i) the adhesion strength and (ii) the bond lifetimes, demonstrating how these two parameters can lead to cell stop in finite time. Finally, we compare our models with experimental data of the literature. 


\section{Linear model}

\subsection{Microscopic linear model}

Let us present the first (linear) microscopic model we consider to describe the convection of a cell by a fluid with ligands binding.

The cell is considered as a point particle, its position at time $t$ is denoted $X_{t}$. The cell is advected by the fluid with a given velocity $u$ in the vicinity of an activated membrane, i.e. surrounded by an array of bond molecules. We restrict our study to the one-dimensional dynamics. We also assume that the Péclet number is large so that the diffusion may be neglected. In such a frame, supposing that the Reynold number and the Stokes number are small, the cell dynamics is non-inertial: the cell has a given drift and is subjected to an active forcing $F_{t}$, which is generated by bonds created between the receptors located on the cell surface and the molecules located on the membrane, see Figure 1. Force balance yields the following equation:

$$
\nu\left(V_{t}-u\right)=F_{t}
$$

where $V_{t}$ is the velocity of the cell and $\nu$ is the friction coefficient (given by Stokes law).

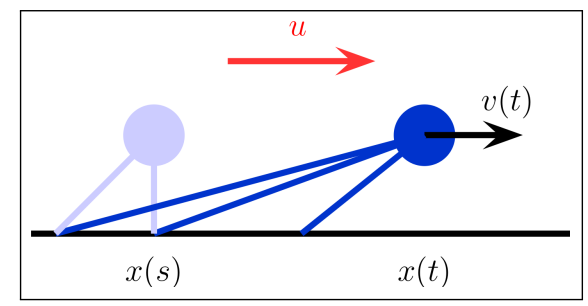

Figure 1: Sketch of the bond binding between the cell and the membrane.

We assume that after an initial binding occurred, each bond exerts a force on the cell. At time $t>s$ the cell occupies position $X_{t}$ and the force exerted by an individual bond created at time $s$ depends on the signed elongation $\left(X_{t}-X_{s}\right)$. This force is supposed to be elastic

$$
f\left(X_{t}, X_{s}\right)=-k\left(X_{t}-X_{s}\right),
$$

where $k$ is the microscopic stiffness coefficient of an individual bond.

However, due to the rupture dynamics of the microscopic bonds some of them may have spontaneously broken. We therefore incorporate a bond 
life-time distribution: the survival probability density on $\mathbb{R}_{+}$is supposed to be given by $g_{\beta}(a):=\beta g(\beta a)$ with $\beta>0$, where $g$ has unit integral and unit first moment:

$$
\int_{\mathbb{R}_{+}} g(a) d a=1, \quad \int_{\mathbb{R}_{+}} a g(a) d a=1 .
$$

Consequently, $1 / \beta$ is the mean bond life-time:

$$
\int_{\mathbb{R}_{+}} a g_{\beta}(a) d a=\frac{1}{\beta} .
$$

More precisely, the bonds dynamics is taken into account in model (1) by assuming that the time distribution of bonds is described by a random measure $M$ on the tensorial time/life-time space $\mathbb{R}_{+} \times \mathbb{R}_{+}$: each cross in Figure 2 (left) represents one bond "activated" at time $s$ and "deactivated" at time $s+a$. The measure $M$ is supposed to be a punctual Poisson process of intensity $\alpha \mathrm{d} s \otimes g_{\beta}(a) \mathrm{d} a$, where $\alpha$ is the bond association rate and $\beta$ is the bond dissociation rate. The two rates are supposed to be constant. A particular attention is paid to the memoryless case, i.e. when $g(a)=$ $\exp (-a)$.
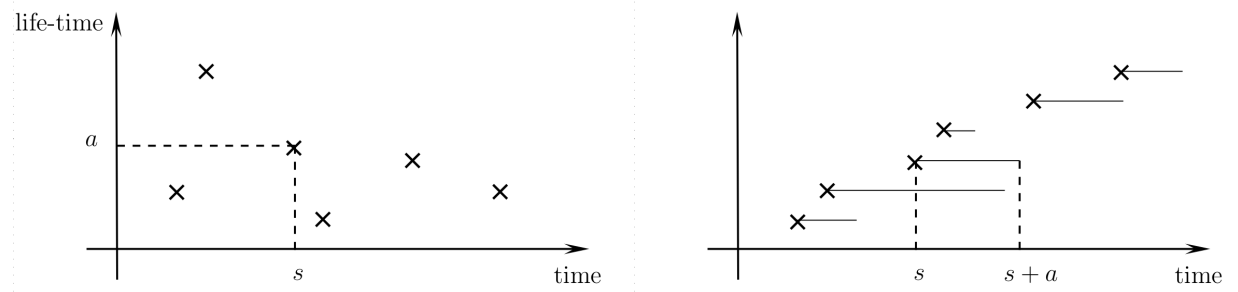

Figure 2: Poisson process on $\mathbb{R}_{+} \times \mathbb{R}_{+}$. Left: crosses represent the random measure on the state-space. Each cross represent one bond. Right: horizontal lines represent the active time of each bond.

Note that the life-time of one bond is supposed to be independent of its individual dynamics. Consequently, the force due to the interaction between the bonds and the driven cell is given by:

$$
F_{t}=\int f\left(X_{t}, X_{s}\right) \mathbf{1}_{0 \leqslant s \leqslant t \leqslant s+a} M(\mathrm{~d} s, \mathrm{~d} a) .
$$

The stochastic linear model is the written

$$
\nu\left(V_{t}-u\right)=-k \int f\left(X_{t}, X_{s}\right) \mathbf{1}_{0 \leqslant s \leqslant t \leqslant s+a} \alpha \mathrm{d} s \otimes g_{\beta}(a) \mathrm{d} a,
$$


which is our reference model. For a more detailed presentation of Poisson processes, we refer to Allen (2011); Méléard (2013).

\subsection{Averaged linear model}

The previous stochastic microscopic model can be averaged to obtain a deterministic one.

We denote $v(t)=\mathbf{E}\left[V_{t}\right]$ and $x(t)=\mathbf{E}\left[X_{t}\right]$ the expectations of the random velocity $V_{t}$ and random position $X_{t}$. They satisfy the following equation:

$$
\nu(v(t)-u)=-k \alpha \int_{0}^{t}(x(t)-x(s)) P_{\beta}(t-s) d s,
$$

where $P_{\beta}(a)=\int_{a}^{+\infty} g_{\beta}(\tau) d \tau$ is the survival probability, i.e. the age distribution of bonds. In the particular (memoryless) case $g_{\beta}(a)=\beta \exp (-\beta a)$, this age distribution is given by $P_{\beta}(a)=\exp (-\beta a)$.

To study this deterministic model, let us introduce dimensionless variables and unknowns, for some reference velocity $U$

$$
t=\tilde{t} / \beta, \quad x(t)=U / \beta \tilde{x}(\tilde{t}), \quad v(t)=U \tilde{v}(\tilde{t}), \quad u=U \tilde{u} .
$$

Omitting the tildes and performing a change of variable, the model becomes the dimensionless equation (5) given below.

Model 1 (Linear elastic model).

$$
\begin{aligned}
& x^{\prime}(t)=v(t), \\
& v(t)=u-\kappa \int_{0}^{t}(x(t)-x(t-a)) P(a) \mathrm{d} a,
\end{aligned}
$$

where $P$ stands for $P_{1}$ and $\kappa=k \alpha /\left(\nu \beta^{2}\right)$ is the unique parameter of the model. Note that $\alpha / \beta$ is the number of bonds in a unit time while $1 / \beta$ is the mean life-time of one bond.

Supposing that the averaged position and velocity are related by (4), equation (4)-(5) is a Volterra integro-differential equation. After integration by parts, it can be written as a linear Volterra equation on the velocity:

$$
v(t)=u-\kappa \int_{0}^{t} v(s)(Q(t)-Q(t-s)) \mathrm{d} s
$$


where $Q$ denotes the anti-derivative of $P: Q^{\prime}=P$ with $Q(0)=0$.

In the case $P(a)=e^{-a}$, we can actually find out the explicit solution:

$$
\begin{aligned}
v(t) & =u\left(1-\kappa A(t) e^{-t}\right) \\
\text { where } \quad A(t) & =\left[\int_{0}^{t}\left(e^{s}-1\right) e^{\kappa\left(s+e^{-s}\right)} d s\right] e^{-\kappa\left(t+e^{-t}\right)} .
\end{aligned}
$$

Asymptotic friction force.. One way to understand the qualitative behavior of this model is to study its long-time asymptotics.

It can be proved that the velocity $v$ is bounded, with $v(t) \in(0, u)$ for all times $t>0$ (see Grec et al. (2017)). Moreover, under some assumptions on $P$, then

$$
v(t) \underset{t \rightarrow+\infty}{\longrightarrow} \frac{u}{1+K}, \quad \text { with } K=\kappa \int_{0}^{+\infty} a P(a) d a .
$$

Thus, the linear elastic microscopic force is asymptotically similar to a friction force of parameter $K$. In particular, this friction force only depends on the second moment of $g_{\beta}$, which is actually the mean bond age. Indeed, we can compute

$$
K=\frac{k \alpha}{\nu} \frac{1}{\beta^{2}} \int_{0}^{+\infty} a P(a) d a=\frac{k \alpha}{2 \nu} \int_{0}^{+\infty} a^{2} g_{\beta}(a) d a
$$

In the case $P(a)=e^{-a}$, we obtain $K=\kappa$, which means that $\lim _{t \rightarrow \infty} v(t)=$ $\frac{u}{1+\kappa}$.

\section{Non-linear models}

In the previous section, we have established that in the long-time limit, the linear elastic microscopic force is similar to a friction force of parameter $K$ which is independent of the shear velocity $u$. Such a result does not agree with in-vitro experiments Munn et al. (1994), which show that the friction force depends on the flow.

In this section we investigate two ways to improve model (1): either by considering a non-linear elastic bond force or by considering force dependent bond rates. Starting from stochastic models, we consider "averaged models" that all lead to a dichotomic asymptotic behavior with different thresholds 
on the convection velocity $u$ in the following form

$$
v(t) \underset{t \rightarrow+\infty}{\longrightarrow} \begin{cases}\frac{u}{1+K(u)}, & \text { if } u \geqslant \bar{u} \\ 0, & \text { if } u \leqslant \bar{u}\end{cases}
$$

This kind of behavior is in accordance with the behavior of slip bonds. We finally perform numerical simulations to assess the validity of these averaged models.

\subsection{Non-linear mechanisms in microscopic models}

Non-linear elastic responses.. A way to take into account the dynamics into the ligand binding is to change the elastic law of the bonds. Indeed, in model 1, the bonds are supposed to behave as linear springs (with Hookean law). In particular, they could extend as long as they do not chemically dissociate. However, since bonds are associations of chemical proteins, we could take into account mechanical rupture events. This is done by assuming that there exists a maximal elongation $\bar{r}>0$ above which bonds break. The linear elastic law with rupture becomes

$$
f_{2}\left(X_{t}, X_{s}\right)=-k\left(X_{t}-X_{s}\right) \mathbf{1}_{\left|X_{t}-X_{s}\right|<\bar{r}},
$$

and the related microscopic model is then written

$$
\nu\left(V_{t}-u\right)=-k \int\left(X_{t}-X_{s}\right) \mathbf{1}_{\left|X_{t}-X_{s}\right|<\bar{r}} \mathbf{1}_{0 \leqslant s \leqslant t \leqslant s+a} M(\mathrm{~d} s, \mathrm{~d} a) .
$$

With such a law, the bonds life-time indirectly depends on the cell dynamics. If the convection velocity is large, ligands rapidly break due to their elongation and the cell might be very weakly slowed down by the ligands. Inversely, if the convection velocity is low enough, the bonds life-time increases and the cell can be drastically slowed down.

Another possibility to model this phenomenon is to impose that the bonds force is bounded: the maximal force exerted by a bond corresponds to the one exerted by one bond of length $\bar{r}>0$. The elastic law becomes

$$
f_{3^{\prime}}\left(X_{t}, X_{s}\right)= \begin{cases}-k \bar{r} \operatorname{sign}\left(X_{t}-X_{s}\right), & \text { if }\left|X_{t}-X_{s}\right|>\bar{r} \\ -k\left(X_{t}-X_{s}\right), & \text { if }\left|X_{t}-X_{s}\right| \leqslant \bar{r}\end{cases}
$$


Here we will study a modified model, where the cut-off part of the elastic law is predominant over the linear part. The elastic behavior is replaced by a constant force model:

$$
f_{3}\left(X_{t}, X_{s}\right)=-k \bar{r} \operatorname{sign}\left(X_{t}-X_{s}\right)
$$

The microscopic models is then written

$$
\nu\left(V_{t}-u\right)=-k \bar{r} \int \operatorname{sign}\left(X_{t}-X_{s}\right) \mathbf{1}_{0 \leqslant s \leqslant t \leqslant s+a} M(\mathrm{~d} s, \mathrm{~d} a) .
$$

Force dependent bond rates. Another approach is to take into account the dependance of bonds association and dissociation rates on the microscopic force exerted on one bond. To this end, we observe that the force exerted by the bonds can be modelled as depending on the velocity of the cell. This viewpoint can be justified by the following approximation for the microscopic elastic force at creation time

$$
f_{4}\left(X_{t}, X_{s}\right)=-k\left(X_{t}-X_{s}\right) \underset{(t-s) \rightarrow 0}{\sim}-k(t-s) V_{t}
$$

Thus, we consider in first approximation that the instantaneous force exerted on one bond is a linear function of the velocity for the whole lifetime of the bond. This leads to introducing the dimensionless quantity $k V_{s} /\left(\beta F_{d}\right)$, where $F_{d}$ is the detachment force (see Korn and Schwarz (2008)). We suppose that each bond created at time $s$ exerts a force proportional to $c\left(k V_{s} /\left(\beta F_{d}\right)\right)$, where $c: \mathbb{R}_{+} \mapsto \mathbb{R}_{+}$is a positive function. Moreover, the life-time of each bond created at time $s$ is given by $A / b\left(k V_{s} /\left(\beta F_{d}\right)\right)$ where the random variable $A$ has $g_{\beta}$ for probability density function and $b: \mathbb{R}_{+} \mapsto \mathbb{R}_{+}$is a positive function of the velocity. Under these assumptions, the microscopic model writes:

$$
\nu\left(V_{t}-u\right)=-k \bar{r} \int\left(X_{t}-X_{s}\right) \mathbf{1}_{0 \leqslant s \leqslant t \leqslant s+a / b\left(k V_{s} / \beta F_{d}\right)} c\left(k V_{s} / \beta F_{d}\right) M(\mathrm{~d} s, \mathrm{~d} a)
$$

If the function $b$ is increasing, the bond life-time decreases as the velocity (and the force) increases: such bonds are called slip bonds. For example, in Bell's model Bell (1978), $b$ is an exponential function of the microscopic force. Conversely, if the function $b$ is decreasing, the bond life-time increases as the velocity (and the force) increases: such bonds are called catch bonds. The bonds involved in the cell adhesion process combine the behaviors of both catch and slip bonds. 


\subsection{Averaged nonlinear models}

Non-linear elastic responses.. Averaging microscopic models (9) or (11), we obtain the following equation:

$$
\nu\left(\mathbf{E}\left[V_{t}\right]-u\right)=-k \int_{0}^{t} \mathbf{E}\left[f\left(X_{t}, X_{s}\right)\right] P_{\beta}(t-s) \alpha \mathrm{d} s .
$$

In the case of non-linear forces, this expression can not be further simplified. However, we suppose that the approximation

$$
\mathbf{E}\left[f\left(X_{t}, X_{s}\right)\right] \approx f\left(\mathbf{E}\left[X_{t}\right], \mathbf{E}\left[X_{s}\right]\right)
$$

is valid and we consider the following deterministic dimensionless models, with the same dimensionless quantities introduced in (3).

Model 2 (Bond maximal elongation).

$$
\begin{aligned}
& x^{\prime}(t)=v(t), \\
& v(t)-u=-\kappa \int_{0}^{t}(x(t)-x(s)) \mathbf{1}_{|x(t)-x(s)|<\rho} P(t-s) d s,
\end{aligned}
$$

where $\kappa=k \alpha /\left(\nu \beta^{2}\right)$ and $\rho=(\beta / U) \bar{r}$ are the two dimensionless parameters.

Model 3 (Constant bond force).

$$
\begin{aligned}
& x^{\prime}(t)=v(t), \\
& v(t)-u=-\kappa \rho \int_{0}^{t} \operatorname{sign}(x(t)-x(s)) P(t-s) d s,
\end{aligned}
$$

where $\kappa \rho=k \alpha \bar{r} /(\nu \beta U)$ is the unique dimensionless parameter.

Force-dependent bond rates.. Taking the expectation of (12), we obtain:

$\nu\left(\mathbf{E}\left[V_{t}\right]-u\right)=\int \mathbf{E}\left[\left(X_{t}-X_{s}\right) c\left(k V_{s} / \beta F_{d}\right) \mathbf{1}_{0 \leqslant s \leqslant t \leqslant s+a / b\left(k V_{s} / \beta F_{d}\right)}\right] g_{\beta}(a) \mathrm{d} a \alpha \mathrm{d} s$.

The non-linearity prevents us again from obtaining a closed deterministic equation. However, we consider the following dimensionless model: 
Model 4 (Velocity-dependent bond rates).

$$
\begin{aligned}
& x^{\prime}(t)=v(t) \\
& v(t)-u=-\kappa \int_{0}^{t}(x(t)-x(s)) c(\phi v(s)) P_{b(\phi v(s))}(t-s) \mathrm{d} s .
\end{aligned}
$$

where $\kappa=k \alpha /\left(\nu \beta^{2}\right)$ and $\phi=k U /\left(\beta F_{d}\right)$ are the two dimensionless parameters.

Note that we recover the same expression of the elastic force as in Preziosi and Vitale (2011), where several functions $b$ are considered.

\subsection{Dichotomic asymptotic behaviors}

Contrary to the linear equation (section 2.2), we are not able to rigorously compute the long-time behavior of the velocity $v$ for the previous models 2-4. However, in some cases, we will see that the three non-linear models lead to a velocity-dependent friction parameter and dichotomic behaviors.

Asymptotic behavior of model 2 (Bond maximal elongation). We provide a first result concerning the asymptotic behavior of model 2 .

Assuming that the velocity $v(t)$ solution to (13) converges towards $v_{\infty}$ as $t$ tends to $+\infty$ and if $P \in L^{1}\left(\mathbb{R}_{+}\right)$, then the asymptotic velocity is solution to the non-linear equation (see proposition Appendix A.1, appendix Appendix A):

$$
v_{\infty}=u-\kappa v_{\infty} \int_{0}^{\rho / v_{\infty}} a P(a) \mathrm{d} a .
$$

In particular, if $a P(a) \in L^{1}$, the limit velocity is non-zero. The large time convergence assumption is not analytically proven but is numerically validated.

Formally, the asymptotic friction coefficient is thus given by:

$$
K=\kappa \int_{0}^{\rho / v_{\infty}} a P(a) \mathrm{d} a .
$$

Contrary to the linear case (see eq. (7)), this coefficient depends non-linearly on the asymptotic velocity $v_{\infty}$ and consequently depends on the convective velocity $u$. To better characterize this non-linearity, we can identify a velocity threshold on $u$ that separates stopping from rolling. Considering the infinite bond life-time limit $\beta \rightarrow+\infty$, which corresponds to $\kappa \rightarrow+\infty$ with $\kappa \rho^{2}=$ 
constant, then, as proved in proposition Appendix A.2 (appendix Appendix A), the asymptotic velocity satisfies the second degree polynomial equation:

$$
0=v_{\infty}^{2}-u v_{\infty}+\frac{\kappa \rho^{2}}{2}
$$

This exactly corresponds to the infinite life-time distribution (case $P(a)=1$ ), which implies in particular that the asymptotic velocity is independent of the age distribution of the bonds in this scaling. In particular, the following dichotomy holds:

$$
v_{\infty}= \begin{cases}\frac{u}{2}\left(1 \pm \sqrt{1-\frac{\bar{u}_{2}^{2}}{u^{2}}}\right) & \text { if } u \geqslant \bar{u}_{2} \\ 0 & \text { if } u<\bar{u}_{2}\end{cases}
$$

where $\bar{u}_{2}=\sqrt{2 \kappa \rho^{2}}$ (the subscript refers to model 2) is the threshold velocity. Note that two asymptotic values are possible in the case $u \geqslant \bar{u}_{2}$. We actually conjecture (by monotony argument) that the right asymptotic velocity is the largest one. The simulations confirm this ansatz (see section 3.4). Note also that the asymptotic behavior is independent of the life-time distribution function $P$ : in the limit we consider, all the life-time distribution can be identified to the infinite life-time distribution since mechanical ruptures happen long before chemical dissociation.

In Figure 3 (left), we compute numerically solutions of the non-linear equation (A.1) (with a Newton method) when $P(a)=e^{-a}$ for several values of $\kappa$, and plot the relative velocity with respect to $u$. We observe a dichotomy behavior for any value of $\kappa$ : for high convective velocity $u$, the bonds friction is not active (cells are weakly slowed down), whereas the friction is much higher for small $u$. When increasing $\kappa$ with $\kappa \rho^{2}=1$, the dichotomy behavior is strengthened. The asymptotic curve when $\kappa \rightarrow+\infty$ is exactly the one obtained for $P(a)=1$. This suggests that the right dichotomic threshold to consider is given by $\bar{u}_{2}=\sqrt{2 \kappa \rho^{2}}$.

Asymptotic behavior of model 3.. To study the asymptotic behavior of model 3 , let us introduced a regularized model:

$$
\begin{aligned}
& x^{\prime}(t)=v(t), \\
& v(t)-u=-\kappa \rho \int_{0}^{t} f_{\varepsilon}(x(t)-x(s)) P(t-s) d s,
\end{aligned}
$$


where the elastic law $f_{\varepsilon}$ is given by

$$
f_{\varepsilon}(\ell)= \begin{cases}\operatorname{sign}(\ell), & \text { if }|\ell|>\varepsilon \\ \ell / \varepsilon, & \text { if }|\ell| \leqslant \varepsilon\end{cases}
$$

The limit $\varepsilon \rightarrow 0$ gives formally the constant force model. Note that taking $\varepsilon=r$, we recover the bounded force model (10). Assuming that the velocity $v(t)$ solution to (18) is positive and converges towards $v_{\infty}$ as $t$ tends to $+\infty$, the asymptotic velocity satisfies the following non-linear equation:

$$
v_{\infty}^{\varepsilon}=u-\kappa \rho \int_{a>\varepsilon / v_{\infty}^{\varepsilon}} P(a) d a-\kappa \rho \frac{v_{\infty}^{\varepsilon}}{\varepsilon} \int_{a<\varepsilon / v_{\infty}^{\varepsilon}} a P(a) d a .
$$

The asymptotics $\varepsilon \rightarrow 0$ gives:

$$
v_{\infty}= \begin{cases}u-\bar{u}_{3}, & \text { if } u>\bar{u}_{3} \\ 0, & \text { if } u<\bar{u}_{3} .\end{cases}
$$

with $\bar{u}_{3}=\kappa \rho$. Note that, as for the previous model, the asymptotic behavior does not depend on the particular life-time distribution $P$.
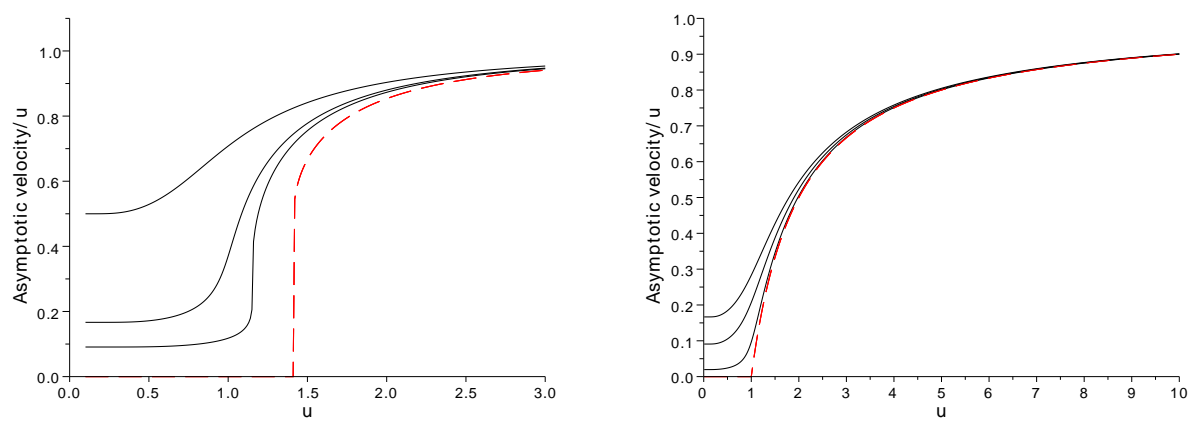

Figure 3: Left (Elastic bond with elongation rupture): Asymptotic velocity $v_{\infty}$ divided by $u$ as a function of the initial velocity $u$ with $\kappa \rho^{2}=1$. Continuous line: Case $P(a)=e^{-a}$ with, from top to down, $\kappa=1, \kappa=5, \kappa=10$. Dashed line: Case $P(a)=1$. Right (Constant bond force): Asymptotic velocity $v_{\infty}$ divided by $u$ as a function of the initial velocity $u$ with $\kappa \rho=1$. In black continuous lines: from top to down, $\varepsilon=0.2, \varepsilon=0.1, \varepsilon=0.02$. In red dashed line: asymptotic $\varepsilon \rightarrow 0$. 
In the particular case where $P(a)=e^{-a}$, equation (19) is written

$$
v_{\infty}^{\varepsilon}=u-\kappa \rho \frac{v_{\infty}^{\varepsilon}}{\varepsilon}\left(1-\exp \left(-\varepsilon / v_{\infty}^{\varepsilon}\right)\right)
$$

In Figure 3 (right), we plot the asymptotic velocity as a function of the convection velocity $u$ by solving (21) (by Newton method) for different values of $\varepsilon$. We compare with the limit behavior (20).

Asymptotic behavior of model 4.. Assuming that the velocity $v(t)$ solution to (14) is positive and converges towards $v_{\infty}$ as $t$ tends to $+\infty$, then the asymptotic velocity satisfies the following non-linear equation (see proposition Appendix A.3, appendix Appendix A) :

$$
v_{\infty}=u-\kappa v_{\infty} c\left(\phi v_{\infty}\right) \int_{0}^{+\infty} a P_{b\left(\phi v_{\infty}\right)}(a) d a .
$$

As for the previous model, these assumptions are not analytically proven but they are numerically validated. For this model, we do not obtain general threshold behavior independent of the life-time distribution. In the particular case $P_{b}(a)=\exp (-b a)$, equation (A.6) becomes:

$$
v_{\infty}=u-\kappa v_{\infty} c\left(\phi v_{\infty}\right) / b\left(\phi v_{\infty}\right)^{2} .
$$

Depending on the rates functions $b$ and $c$, we can obtain the same asymptotic behavior as the previous models.

- For $c(\phi v)=1, b(\phi v)=\phi v$, we recover the same dichotomic asymptotic behavior as for model 2 (maximal elongation) but with threshold velocity

$$
\bar{u}_{4}=2 \sqrt{\kappa} / \phi .
$$

- Similarly, for $c(\phi v)=\phi v, b(\phi v)=\phi v$, we now recover a similar dichotomic asymptotic behavior for model 3 (constant force) with threshold velocity

$$
\bar{u}_{5}=\kappa / \phi .
$$

Both previous cases would correspond to slip bonds since the dissociation rate increases as the microscopic force increases. 
- This model can also be used to describe catch bonds. Choosing $c(\phi v)=$ 1 and $b(\phi v)=1 / \sqrt{\phi v+1}$, the only positive solution of equation (A.7) is given by

$$
\begin{aligned}
v_{\infty} & =\frac{\sqrt{(\kappa+1)^{2}+4 \kappa \phi u}-(\kappa+1)}{2 \kappa \phi} \\
& =\frac{u}{(\kappa+1)} \frac{2}{\left(\sqrt{1+4 \kappa \phi u /(\kappa+1)^{2}}+1\right)} .
\end{aligned}
$$

The relative damped velocity $v_{\infty} / u$ is thus a decreasing function with respect to the drift velocity $u$, due to the fact that catch bonds becomes more resistant when submitted to larger stress. Note that for low value of drift velocity $u$, we recover the damping factor of the linear model (4)-(5).

\subsection{Numerical simulations}

In this section, we compare the averaged models with the underlying stochastic dynamics. We also illustrate numerically that the models indeed converge to the formal asymptotic behaviors obtained in the previous section, and check the positivity and convergence assumptions made previously.

Maximal elongation model.. Although it is not possible to prove mathematically the convergence of (9) towards Model 2, numerical simulations indicate that the two models behave analogously. On Figure 4, we consider the case $P(a)=e^{-a}$ and we plot two different simulations of the microscopic model, compared with the deterministic one, with $\kappa=0.7, u=1, \rho=5$, in which case $\bar{u}_{2} \simeq 5.92$. Then, $u<\bar{u}_{2}$, and we indeed observe that the asymptotic velocity is much smaller than $u=1$. This somehow confirms that $\bar{u}_{2}$ could be the right threshold to consider. We see that the asymptotic behavior of both microscopic and averaged models is the same. Moreover, it converges towards the asymptotic value predicted by the computations (solution of (A.1)).

In order to numerically check the convergence, we computed the average of the results of 40 simulations. This average is compared with the simulation of the deterministic model (Figure 5 (right)). Moreover, we also plotted the relative $L^{2}(0, T)$-norm of the difference between the deterministic model and this microscopic average as a function of $i$, which is given on Figure 5 (left).

We can also perform the same numerical verification in the case of an infinite life-time distribution (case $P(a)=1$ ). In this case, we recover the 


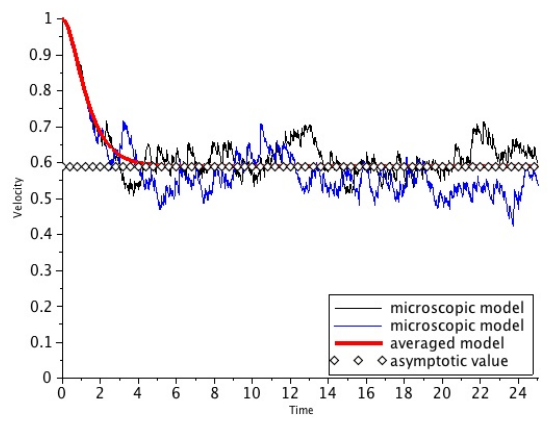

Figure 4: (Maximal elongation model) Case $P(a)=e^{-a}$. Velocity $v$ as a function of time using the microscopic model (9) and the deterministic model 2. Diamond line: Asymptotic value computed by (A.1).
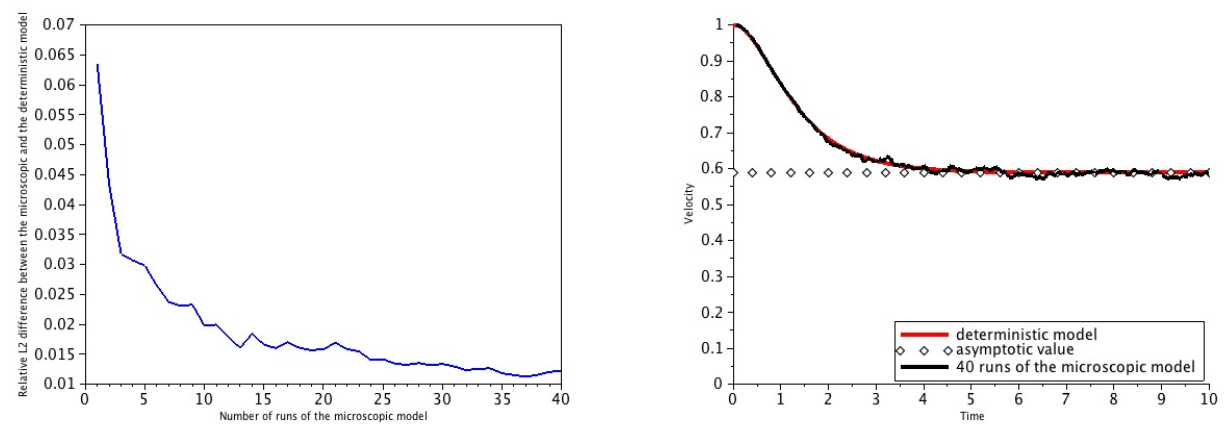

Figure 5: (Maximal elongation model) Case $P(a)=e^{-a}$. Left: Relative $L^{2}$-error between the deterministic model and the average of $i$ runs of the microscopic one. Right: Comparison between the velocity of the deterministic model and the average of 40 runs of the microscopic one.

dichotomy described (A.3) depending on the value of $u$. In particular, if we choose $u<\bar{u}_{2}=\sqrt{2 \kappa \rho^{2}}$, the velocity converges to zero, whereas for $u>\bar{u}_{2}$, it converges towards a non-zero value given by the formula (A.3) (see Figure 6 with the following values for the parameters $\kappa=0.7, \rho=5$ and $u=5$ (left) or $u=7$ (right), since $\bar{u}_{2} \simeq 5.92$ ).

Constant bond force. In a similar way, we can consider the model of a constant force and compare again its microscopic and deterministic versions. 

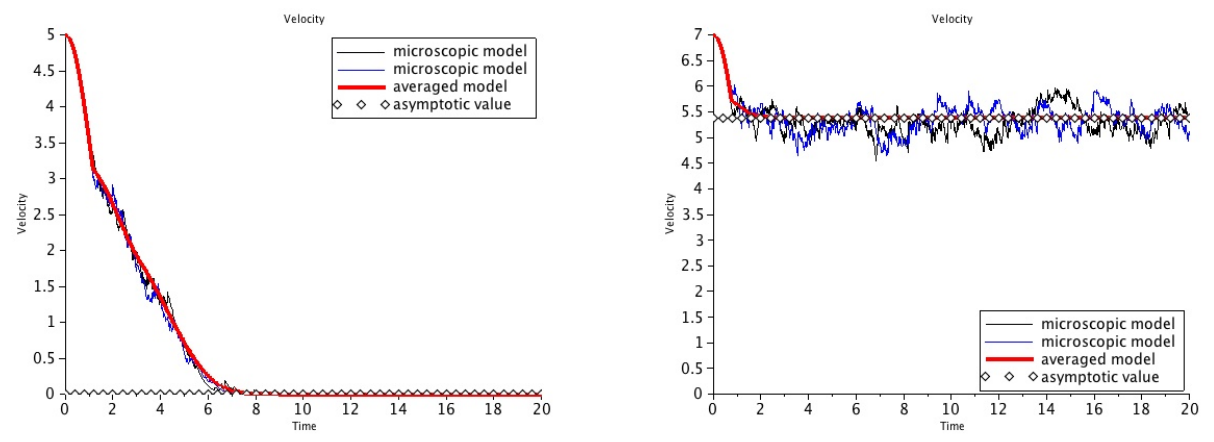

Figure 6: (Maximal elongation model) Case $P(a)=1$. Velocity $v$ as a function of time using the microscopic model (9) and the deterministic model 2. Diamond line: Asymptotic value computed by (A.3). Left: $u<\bar{u}_{2}=\sqrt{2 \kappa \rho^{2}}$. Right: $u>\bar{u}_{2}$.

This is done on Figure 7, with $\bar{u}_{3}=\kappa \rho=0.7$, for two different values $u=2>\bar{u}_{3}$ (thus $u-\bar{u}_{3}=1.3$ ) and $u=0.6<\bar{u}_{3}$. Again, we see that the deterministic model has the same behavior as the microscopic one. Moreover, we recover the asymptotic dichotomic behavior with the right values predicted by the theory in the case $\varepsilon \rightarrow 0$ (equation (20)). The velocity is highly oscillating as soon as it vanishes for the first time, which is the case when $u<\bar{u}_{3}$. However, the corresponding position is almost constant due to oscillation balancing.

This model can be approximated by the regularized one introduced in (18), for which we obtain the same asymptotic behaviors as long as $\varepsilon$ is small enough. Of course, for a fixed non zero value of $\varepsilon$, the asymptotic value of (18) when $u<\bar{u}_{3}$ is not exactly zero, but it converges to zero when $\varepsilon$ tends to zero. Therefore, the dichotomic behavior is only true in the limit $\varepsilon \rightarrow 0$. Numerical simulations also confirm these results.

Force dependent bond rates. For the velocity-dependent bond rate model, we consider several functions $b$ and $c$ as in Section 3.3.

- On Figure 8, we consider the case $c(\phi v)=1$ and $P_{b}(a)=e^{-b a}$ with $b(\phi v)=v$ (slip bonds). We plot again two different simulations of the microscopic model, compared with the deterministic one, with $\kappa=0.7$, $u=1<\bar{u}_{4}$ (left) or $u=2>\bar{u}_{4}$ (right) ( since $\bar{u}_{4}=2 \sqrt{\kappa} \simeq 1.67$ ). We see once again that the long-time behavior is the same. Moreover, it converges towards the asymptotic value predicted by the computations 

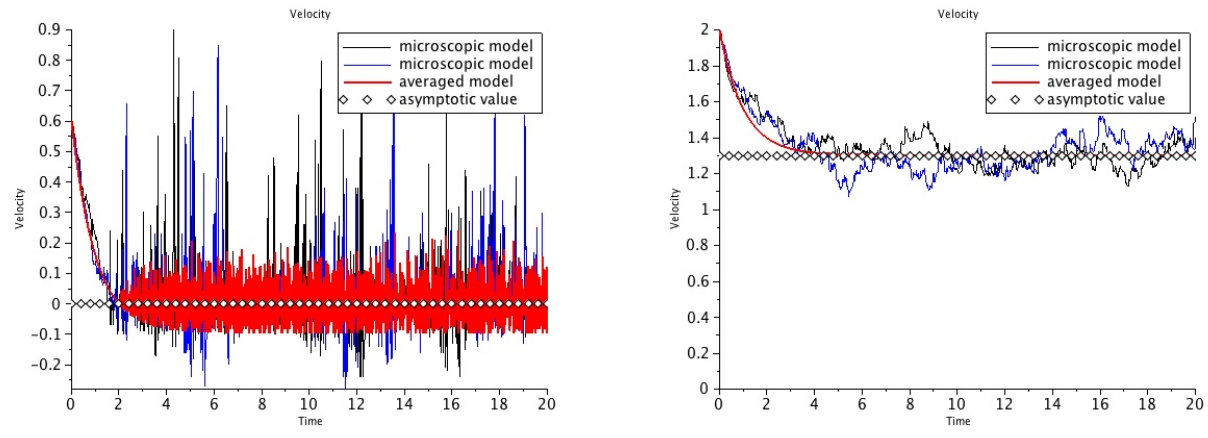

Figure 7: (Constant bond force) Velocity $v$ as a function of time using the microscopic model (11) and the deterministic model 3. Diamond line: Asymptotic value. Left: $u<\bar{u}_{3}$. Right: $u>\bar{u}_{3}$.

(solution of (A.6)). We observe on Figure 8 (left) that although the velocity tends to zero for large time, the convergence to zero is much slower than for the case of the bond maximal elongation of the bonds (Figure 6).
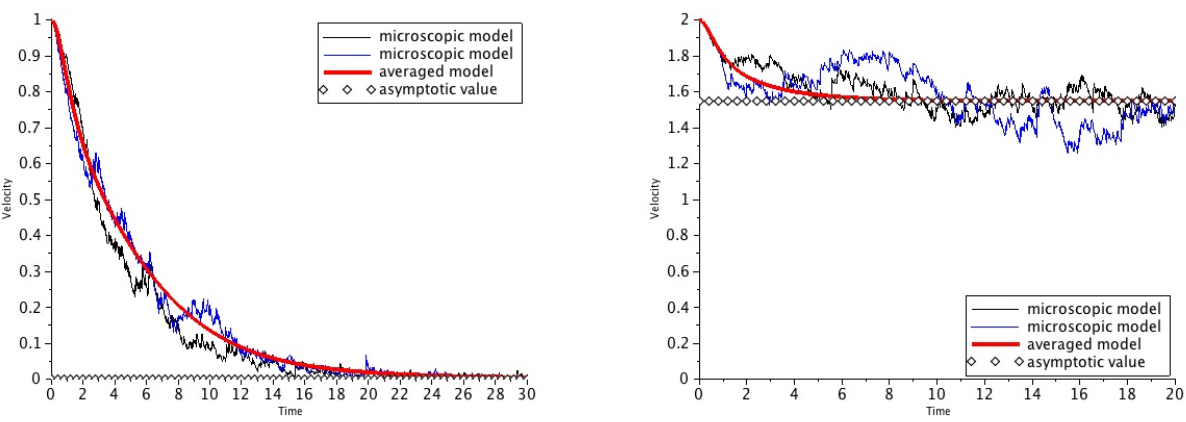

Figure 8: (Velocity dependent bond rates) Case $b(\phi v)=v$ and $c(\phi v)=1$. Velocity $v$ as a function of time using the microscopic model (12) and the deterministic model 4. Diamond line: Asymptotic value. Left: $u<\bar{u}_{4}$. Right: $u>\bar{u}_{4}$.

- The same is observed for the case $c(\phi v)=1$ and $P_{b}(a)=e^{-b a}$ with $b(\phi v)=1 / \sqrt{v+1}$ (catch bonds), where we recover the value $v_{\infty}$ given by (24) (see Figure 9, with the parameters $\kappa=0.7$ and $u=2$ ). 


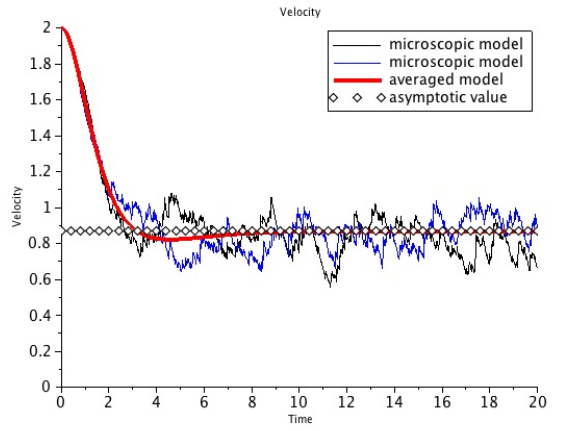

Figure 9: (Velocity dependent bond rates) Case $b(\phi v)=1 / \sqrt{v+1}$ and $c(\phi v)=$ 1. Velocity $v$ as a function of time using the microscopic model (12) and the deterministic model 4. Diamond line: Asymptotic value computed by (24).

We also note that in the case of catch bonds, the velocity of the deterministic model is not monotonic. Numerically, if the velocity $v$ equals $v_{\infty}$ at some time $t$, it does not ensure that it remains equal to $v_{\infty}$.

\section{Discussion and comparison with experimental data}

In this discussion, we compare the theoretical friction laws (7) and (8) obtained asymptotically for the linear and non-linear models with experimental data mostly taken from Alon et al. (1997); Korn and Schwarz (2008). More precisely, we discuss how the linear and non-linear models introduced in previous sections could match experimental data.

In vitro experiments have been carried out in order to measure the friction force induced by different ligands and the resulting rolling velocity. In Alon et al. (1997), the authors consider neutrophils rolling on a P-selectin coated membrane. Ligands P-selectin on the leukocyte surface binds to receptors PSGL1 (P-selectin glycoprotein ligand-1) on the membrane. The different parameters of the experiment are reported in Table 1.

Let us make some comments on these parameters.

Bonds are located at the end of microvilli which are large (up to $4 \mu \mathrm{m}$ ) compared to the ligand-receptor complex. The "ligand stiffness" is here the stiffness of the association of the microvilli and the ligand-receptor. For estimating the ligand creation rate, we determine the number of ligands present in the contact zone: in Korn and Schwarz (2008), this radius of the 


\begin{tabular}{|c|c|c|c|}
\hline Parameters & Symbol & Typical Value & Ref. \\
\hline Leucocyte radius & $R$ & $4 \mu \mathrm{m}$ & $\begin{array}{l}\text { Bell } \\
\text { (1978)Alon } \\
\text { et al. (1997) }\end{array}$ \\
\hline Blood dynamic viscosity & $\eta$ & $1 \mathrm{mPa} \mathrm{s}$ & $\begin{array}{l}\text { Korn and } \\
\text { Schwarz (2008) }\end{array}$ \\
\hline $\begin{array}{l}\text { Flow friction coefficient } \\
\text { (Stokes Law) }\end{array}$ & $\nu=6 \pi \eta R$ & $\begin{array}{l}7.5 \times 10^{-8} \mathrm{~kg} \\
\mathrm{~s}^{-1}\end{array}$ & \\
\hline Ligand stiffness & $k$ & $\begin{array}{l}4.3 \times 10^{-5} \mathrm{~N} \\
\mathrm{~m}^{-1}\end{array}$ & $\begin{array}{l}\text { Korn and } \\
\text { Schwarz (2008) }\end{array}$ \\
\hline Ligand dissociation rate & $\beta$ & $1 \mathrm{~s}^{-1}$ & $\begin{array}{l}\text { Alon et al. } \\
(1997)\end{array}$ \\
\hline $\begin{array}{l}\text { Ligand density on the } \\
\text { wall }\end{array}$ & $c_{w}$ & $30 \mu \mathrm{m}^{-2}$ & $\begin{array}{l}\text { Alon et al. } \\
(1997)\end{array}$ \\
\hline $\begin{array}{l}\text { Number of receptor on } \\
\text { the leukocyte }\end{array}$ & $N_{r}$ & $10^{3}$ & $\begin{array}{l}\text { Korn and } \\
\text { Schwarz (2008) }\end{array}$ \\
\hline $\begin{array}{l}\text { Receptor density on the } \\
\text { leukocyte }\end{array}$ & $\begin{array}{l}c_{\text {leuk }}= \\
N_{r} /\left(4 \pi R^{2}\right)\end{array}$ & $4.9 \mu \mathrm{m}^{-2}$ & $\begin{array}{l}\text { Alon et al. } \\
(1997)\end{array}$ \\
\hline Contact radius & $r_{0}=10^{-2} R$ & $40 \mathrm{~nm}$ & $\begin{array}{l}\text { Korn and } \\
\text { Schwarz (2008) }\end{array}$ \\
\hline $\begin{array}{l}\text { Number of ligands in } \\
\text { contact zone }\end{array}$ & $\begin{array}{l}N \\
\pi r_{0}^{2} c_{\text {leuk }}\end{array}=$ & $2.5 \times 10^{-2}$ & \\
\hline Ligand association rate & $k_{o n}$ & $10^{3} \mathrm{~s}^{-1}$ & $\begin{array}{l}\text { Korn and } \\
\text { Schwarz (2008) }\end{array}$ \\
\hline Effective creation rate & $\alpha=N k_{o n}$ & $25 \mathrm{~s}^{-1}$ & \\
\hline $\begin{array}{l}\text { Dimensionless effective } \\
\text { friction parameter in } \\
\text { model } 1\end{array}$ & $\begin{array}{l}\kappa \\
k \alpha /\left(\nu \beta^{2}\right)\end{array}$ & 14000 & \\
\hline
\end{tabular}

Table 1: Parameters for leukocytes in flow chamber experiment: neutrophil rolling on P-selectin coated membrane and P-selectin binds PSGL1.

contact zone is estimated to be one hundredth of the leucocyte radius. Since the receptor density on the leukocyte is smaller than the ligand density on the wall, we consider that the receptor density on the leukocyte is relevant. From the concentration of receptors $c_{\text {leuk }}$ and the chemical association rate 
$k_{\text {on }}$, we obtain the effective creation rate $\alpha$. The dissociation rate is measured from experiments Alon et al. (1997). We finally obtain a friction parameter equal to $\kappa=14000$.

From the linear model 1, the rolling velocity is interpreted as the non-zero asymptotic value of the velocity. It is thus expected to be equal to $u /(1+\kappa)$, where $u$ is the convective velocity of flow near the endothelial wall. The convective velocity $u$ can be expressed as a function of the shear rate $\dot{\gamma}$, by making the following assumption:

$$
u=R \dot{\gamma}
$$

More precise approximations can be found in Hammer and Lauffenburger (1987); Goldman et al. (1967). In Table 2, we first provide the rolling velocity for the linear model for several values of the shear stress varying from 0.5 to 1.5 dyn $\mathrm{cm}^{-2}$. The rolling velocity is of order $10^{-2} \mu \mathrm{m} \mathrm{s}^{-1}$ : neutrophils are nearly stopped. This is not in agreement with results of (Alon et al., 1997, Figure 1 - bottom), where the asymptotic velocity is of order $50 \mu \mathrm{m}$ $\mathrm{s}^{-1}$. Consequently, this confirms that the linear model does not seem to be precise enough to capture the right behavior.

\begin{tabular}{llll}
\hline Parameters & Symbol & Range & Ref. \\
\hline Shear rate & $\dot{\gamma}$ & $50-150 \mathrm{~s}^{-1}$ & $\begin{array}{l}\text { Korn } \\
\text { and } \\
\text { Schwarz } \\
(2008)\end{array}$ \\
Shear stress & $\tau=\eta \dot{\gamma}$ & $0.5-1.5 \quad \mathrm{dyn}$ & \\
Convective velocity & $u \approx R \dot{\gamma}$ & $200-600 \mu \mathrm{m} \mathrm{s}^{-1}$ & \\
Drag force & $\nu u$ & $14-42 \mathrm{pN}^{-2}$ & \\
\hline $\begin{array}{l}\text { Rolling velocity for the } \\
\text { linear model }\end{array}$ & $u /(1+\kappa)$ & $1.4-4 \times 10^{-2} \mu \mathrm{m}$ & \\
\hline
\end{tabular}

Table 2: Flow parameters for leukocytes in flow chamber experiment and rolling velocity obtained by model 1 .

In Table 3, we also compute the threshold velocities obtained for the different non-linear models. We note that only the maximal elongation model 


\begin{tabular}{|c|c|c|c|}
\hline Parameters & Symbol, Formula & Range & Ref. \\
\hline $\begin{array}{l}\text { Maximum microvilli } \\
\text { length }\end{array}$ & $\bar{r}$ & $1-4 \mu \mathrm{m}$ & $\begin{array}{l}\text { Alon } \\
\text { et al. } \\
(1997)\end{array}$ \\
\hline \multirow[t]{2}{*}{ Detachment force } & $F_{d}$ & $200 \mathrm{pN}$ & $\begin{array}{l}\text { Korn } \\
\text { and } \\
\text { Schwarz } \\
(2008)\end{array}$ \\
\hline & $\phi / U$ & $215 \times 10^{3} \mathrm{~m} \mathrm{~s}^{-1}$ & \\
\hline Threshold in model 2 & $\begin{array}{l}\bar{u}_{2} U=\sqrt{2 \kappa} \rho U= \\
\sqrt{2 \kappa} \bar{r} \beta\end{array}$ & $170-677 \mu \mathrm{m} \mathrm{s}^{-1}$ & \\
\hline Threshold in model 3 & $\bar{u}_{3} U=\kappa \rho U=\kappa \bar{r} \beta$ & $14-56 \mathrm{~mm} \mathrm{~s}^{-1}$ & \\
\hline $\begin{array}{l}\text { Threshold in model } 3 \\
(c(v)=1, b(v)=v)\end{array}$ & $\bar{u}_{4} U=2 \sqrt{\kappa} U / \phi$ & $1.1 \mathrm{~mm} \mathrm{~s}^{-1}$ & \\
\hline $\begin{array}{l}\text { Threshold in model } 4 \\
(c(v)=v, b(v)=v)\end{array}$ & $\bar{u}_{4} U=\kappa U / \phi$ & $65 \mathrm{~mm} \mathrm{~s}^{-1}$ & \\
\hline $\begin{array}{l}\text { Rolling velocity in model } \\
2 \text { with } P=1\end{array}$ & $\frac{u}{2}\left(1+\sqrt{1-\frac{\bar{u}_{2}}{u^{2}}}\right)$ & $100-588 \mu \mathrm{m} \mathrm{s}^{-1}$ & \\
\hline $\begin{array}{l}\text { Rolling velocity in } \\
\text { model } 3 \text { or } 4\end{array}$ & $0\left(\right.$ since $\left.u<\bar{u}_{i} U\right)$ & $0 \mu \mathrm{m} \mathrm{s}^{-1}$ & \\
\hline
\end{tabular}

Table 3: Flow parameters for leukocytes in flow chamber experiment and rolling velocities for the different nonlinear models.

2 gives a threshold velocity in the range of the typical flow velocity. We can then compute the corresponding rolling velocity, which gives at least a better order of magnitude than the linear model. Nevertheless, the value of the threshold is highly dependent on the parameters of the model, e.g. the value of the maximal length of microvilli. The value of the rolling velocity can only be captured by this model 2 ; in other models, the threshold velocity being too high $\left(u<\bar{u}_{3}\right.$ and $\left.u<\bar{u}_{4}\right)$, the asymptotic velocity is zero.

In order to compare more precisely with experimental values given in (Alon et al., 1997, Figure 1 - bottom), we choose the fluid velocity to be $u=$ $650 \mu \mathrm{m} \mathrm{s}^{-1}$. The rolling velocity given by model 2 in the case $P=1$ is at least $\simeq 340 \mu \mathrm{m} \mathrm{s}^{-1}$ (obtained for $\bar{r} \simeq 4 \mu \mathrm{m}$ ). This value is largely overestimated 
when comparing to experimental data. Moreover, we see that the predicted asymptotic velocity given by (A.3) always remains in the interval $\left[\frac{u}{2} ; u\right]$, thus it cannot be expected to reproduce a rolling velocity $v_{\infty} \simeq 30 \mu \mathrm{m} \mathrm{s}^{-1} \simeq 0.04 u$.

In order to recover such values, model 2 has to be considered with a finite lifetime distribution, i.e. in the case $P$ non constant, for example $P(a)=$ $\exp (-\beta a)$. Then, the solution to (A.1) is not explicit anymore but can be computed numerically. We observe that $v_{\infty} \simeq 30 \mu \mathrm{m} \mathrm{s}^{-1}$ corresponds to a microvilli length equals to $\bar{r} \simeq 13 \mu \mathrm{m}$. This value is of the right order of magnitude, but slightly overestimated.

Another parameter for which the values given in Table 1 can have some variability is the chemical association rate $k_{\text {on }}$. Again, the solution to (A.1) is computed numerically. We observe that $v_{\infty} \simeq 30 \mu \mathrm{m} \mathrm{s}^{-1}$ corresponds to an association rate equals to $k_{o n}=1.1 \times 10^{4} \mathrm{~s}^{-1}$, which remains in the order of magnitude given in Korn and Schwarz (2008).

Moreover, it has been observed in the literature that the velocity of a neutrophil depends strongly on its deformability Dong and Lei (2000); Czerwinska et al. (2017), which could also explain the variability for fitting the parameters.

To conclude, parameter estimation shows that the linear model overestimates the friction force. Non-linear models could provide some hints for studying the impact of the convective flow on adhesion. Specific experiments could be carried out to validate or invalidate the different hypotheses. Note also that the threshold velocities are specific for the chemical bonds: therefore, this could explain the role of the different ligands involved in the adhesion cascade (selectin, integrin) Ley et al. (2007).

In addition, similarly as previous models for leukocyte adhesion Korn and Schwarz (2008), this type of simple models with very few parameters could also be used in other types of applications, e.g. adsorption of a homopolymeric gobule (see for example Radtke and Netz (2015)), or glycomechanics of the metastatic cascade (see for example Geng et al. (2012)).

\section{Appendix A. Proof of the dichotomic asymptotic behaviors of mod- els 2 and 4}

Proposition Appendix A.1 (Asymptotic behavior of model 2). Assuming that the velocity $v(t)$ solution to (13) converges towards $v_{\infty}$ as $t$ tends to $+\infty$ and if $P \in L^{1}\left(\mathbb{R}_{+}\right)$, then the asymptotic velocity is solution to the non-linear 
equation:

$$
v_{\infty}=u-\kappa v_{\infty} \int_{0}^{r / v_{\infty}} a P(a) \mathrm{d} a .
$$

In particular, if aP $(a) \in L^{1}$, the limit velocity is non-zero.

Proof. Assuming that $v(t)$ converges to $v_{\infty}$, then we see that

$$
\mathbf{1}_{|x(t)-x(t-a)|<r}(x(t)-x(t-a)) P(a) \rightarrow \mathbf{1}_{v_{\infty} a<r} v_{\infty} a P(a) .
$$

Since $\mathbf{1}_{|x(t)-x(t-a)|<r}(x(t)-x(t-a)) P(a)<r P(a) \in L^{1}$, the dominated convergence theorem implies the first result.

Proposition Appendix A.2 (Asymptotic behavior of model 2, limit $\beta \rightarrow+\infty$ ). Considering the limit $\kappa \rightarrow+\infty$ with $\kappa r^{2}=$ constant, then the asymptotic velocity satisfies the second degree polynomial equation:

$$
0=v_{\infty}^{2}-u v_{\infty}+\frac{\kappa r^{2}}{2}
$$

This exactly corresponds to the infinite life-time distribution (case $P(a)=1$ ). In particular, the following dichotomy holds:

$$
v_{\infty}= \begin{cases}\frac{u}{2}\left(1 \pm \sqrt{1-\frac{\bar{u}_{2}^{2}}{u^{2}}}\right) & \text { if } u \geqslant \bar{u}_{2}, \\ 0 & \text { if } u<\bar{u}_{2}\end{cases}
$$

where $\bar{u}_{2}=\sqrt{2 \kappa r^{2}}$ (the subscript refers to model 2).

Proof. Starting from (A.1), we have:

$$
\begin{aligned}
v_{\infty} & =u-\kappa r^{2} v_{\infty} \int_{0}^{r / v_{\infty}} \frac{a}{r} P(a) \frac{\mathrm{d} a}{r} \\
& =u-\kappa r^{2} v_{\infty} \int_{0}^{1 / v_{\infty}} s P(r s) \mathrm{d} s
\end{aligned}
$$

Taking the limit $r \rightarrow 0$ while keeping $\kappa r^{2}$ constant, we obtain equation (A.2) since $P(0)=1$.

In the case $P(a)=1$, the asymptotic velocity satisfies

$$
v_{\infty}=u-\kappa v_{\infty} \int_{0}^{r / v_{\infty}} a \mathrm{~d} a
$$


which also turns into equation (A.2).

This second order equation has real solutions only if its discriminant is positive, which provides the condition

$$
u>\bar{u}_{2}=\sqrt{2 \kappa r^{2}} .
$$

Under this condition, the dynamics has two non-zero positive asymptotic velocities given by

$$
v_{\infty}^{ \pm}=\frac{u}{2}\left(1 \pm \sqrt{1-\frac{\bar{u}_{2}^{2}}{u^{2}}}\right) .
$$

Suppose $u<\bar{u}_{2}$. Since (A.2) has no real solution, the integral has to be non definite, which implies $v_{\infty}=0$.

Proposition Appendix A.3 (Asymptotic behavior of model 4). Assuming that the velocity $v(t)$ solution to $(14)$ is positive and converges towards $v_{\infty}$ as $t$ tends to $+\infty$, then the asymptotic velocity satisfies the following non-linear equation:

$$
v_{\infty}=u-\kappa v_{\infty} c\left(\phi v_{\infty}\right) \int_{0}^{+\infty} a P_{b\left(\phi v_{\infty}\right)}(a) d a .
$$

Proof. Assuming that $v(t)$ converges to $v_{\infty}>0$, then we see that

$$
(x(t)-x(t-a)) c(\phi v(t-a)) P_{b(\phi v(t-a))}(a) \rightarrow v_{\infty} a c\left(\phi v_{\infty}\right) P_{b\left(\phi v_{\infty}\right)}(a) .
$$

Since $|(x(t)-x(t-a))| P(a, v(t-a))<v_{M} a P\left(a, v_{m}\right) \in L^{1}$, where $0<v_{m}<$ $v(t)<v_{M}$ are lower and upper bounds of $v(t)$, we can apply the dominated convergence theorem. We obtain:

$$
v_{\infty}=u-\kappa v_{\infty} c\left(\phi v_{\infty}\right) \int_{0}^{+\infty} a P_{b\left(\phi v_{\infty}\right)}(a) d a .
$$

\section{Bibliography}

Allen, L. J. S., 2011. An introduction to stochastic processes with applications to biology, 2nd Edition. CRC Press, Boca Raton, FL.

Alon, R., Chen, S., Puri, K. D., Finger, E. B., Springer, T. A., 1997. The Kinetics of L-selectin Tethers and the Mechanics of Selectin-mediated Rolling. The Journal of Cell Biology 138 (5), 1169-1180.

URL http://jcb.rupress.org/content/138/5/1169. abstract 
Bell, G., 1978. Models for the specific adhesion of cells to cell. Science 200, 618-627.

Bell, G., Dembo, M., Bongrand, P., 1984. Competition between nonspecific repulsion and specific bonding. Biophys. J. 45, 1051-1064.

Czerwinska, J., Pumpurus, L., Rieger, M., Uehlinger, D., Nita, I., Blank, F., 2017. Mobility and shape adaptation of neutrophil in the microchannel flow. Journal of the Mechanical Behavior of Biomedical Materials 69, 294300 .

Dong, C., Lei, X. X., 2000. Biomechanics of cell rolling: shear flow, cellsurface adhesion, and cell deformability. Journal of biomechanics 33 (1), $35-43$.

Finger, E., Puri, K., Alon, R., Lawrence, M., von Andrian, U., Springer, T., 1996. Adhesion through L-selectin requires a threshold hydrodynamic shear. Nature 379, 266-269.

Geng, Y., Marshall, J. R., King, M. R., 2012. Glycomechanics of the metastatic cascade: tumor cell-endothelial cell interactions in the circulation. Annals of biomedical engineering 40 (4), 790-805.

Goldman, A., Cox, R., Brenner, H., 1967. Slow viscous motion of a sphere parallel to a plane wall II: Couette flow. Chemical Engineering Science $22(4), 653-660$.

Grec, B., Maury, B., Meunier, N., Navoret, L., 2017. Large time asymptotic of an integro-differential model for cell adhesion, in preparation.

Hammer, D., Apte, S., 1992. Simulation of cell rolling and adhesion on surfaces in shear flow: general results and analysis of selectin-mediated neutrophil adhesion. Biophysical Journal 63 (1), 33-57.

Hammer, D., Lauffenburger, D., 1987. A dynamical model for receptormediated cell adhesion to surfaces. Biophys. J. 52, 475-487.

Helms, G., Dasanna, A. K., Schwarz, U. S., Lanzer, M., 2016. Modeling cytoadhesion of plasmodium falciparum-infected erythrocytes and leukocytes - common principles and distinctive features. FEBS letters 590 (13), 1955-1971. 
Korn, C., 2007. Stochastic dynamics of cell adhesion in hydrodynamic flow. Ph.D. thesis, Universitat Potsdam.

Korn, C. B., Schwarz, U. S., 2008. Dynamic states of cells adhering in shear flow: From slipping to rolling. Phys. Rev. E 77, 041904.

Ley, K., Laudanna, C., Cybulsky, M. I., Nourshargh, S., 2007. Getting to the site of inflammation: the leukocyte adhesion cascade updated. Nature Reviews Immunology 7, 678-689.

Méléard, S., 2013. Processus de branchement. Applications en écologie.

Milisic, V., Oelz, D., 2011. On the asymptotic regime of a model for friction mediated by transient elastic linkages. J. Math. Pures Appl. 96, 484-501.

Munn, L., Melder, R., Jain, R., 1994. Analysis of cell flux in the parallel plate flow chamber: Implications for cell capture studies. Biophysical Journal 67, 889-895.

Oelz, D., Schmeiser, C., 2010. How do cells move? mathematical modelling of cytoskeleton dynamics and cell migration. in Cell mechanics: from single scale-based models to multiscale modelling, eds. A. Chauviere, L. Preziosi, and C. Verdier, Chapman and Hall / CRC Press.

Preziosi, L., Vitale, G., 2011. Mechanical aspects of tumour growth: Multiphase modelling, adhesion, and evolving natural configurations. in M. Ben Amar, A. Goriely, M. M. Müller, L. F. Cugliandolo, Eds., New Trends in the Physics and Mechanics of Biological Systems, p. 177-228, Lecture Notes of the Les Houches Summer School, vol. 92, Oxford University Press.

Radtke, M., Netz, R. R., 2015. Shear-enhanced adsorption of a homopolymeric globule mediated by surface catch bonds. The European Physical Journal E 38 (6), 1-11.

Schwarz, U., Alon, R., 2004. L-selectin-mediated leukocyte tethering in shear flow is controlled by multiple contacts and cytoskeletal anchorage facilitating fast rebinding events. Proceedings of the National Academy of Sciences of the United States of America 5 (18), 6940-6945.

Shamri, R., Grabovsky, V., Gauguet, J.-M., Feigelson, S., Manevich, E., Kolanus, W., Robinson, M. K., Saunton, D. E., von Andrian, U. H., Alon, 
R., 2005. Lymphocyte arrest requires instantaneous induction of an extended lfa-1 conformation mediated by endothelium-bound chemokines. Nature Immunology 5 (6), 497-505. 